dals es ein präformierter, d. h. naturlicher Bestandteil der flores Koso ist.

2. Das konstante Auftreten des Isobuttersäuregeruchs und reichlicher Mengen amorpher rotbrauner Nebenprodukte sprechen dafü, dafs die chemische Umwandlung des Kosotoxins in Kosin kein sehr einfacher Vorgang ist, wie denn auch vor der Hand der Vergleich der elementaren Zusammensetzung und der Molekulargewichte beider Körper einfache Beziehungen derselben, wie z. B. die eines Hydrates zu einem Anhydrid nicht erkennen lassen.

Die Untersuchung des Kosotoxins in chemischer und pharmakologischer Beziehung wird im hiesigen pharmakologischen Institute fortgesetzt.

L $\theta$ ipzig im Dezember 1893.

\title{
Zur Darstellung der Glyoxylsäure.
}

Von Dr. Carl Boettinger.

(Eingegangen den 14. 1. 1894)

Wenn man das von Debus (Liebig's Annalen 100. 1, Kekulé Band II. Seite 20) stammende Rezept kritisch pruft - $220 \mathrm{~g}$. Alkohol von $80 \mathrm{pCt}$. werden mit $100 \mathrm{~g}$. Wasser, dieses mit $200 \mathrm{~g}$. rother rauchender Sulpetersäure in hohen schmalen Flaschen unterschichtet - so muss man sich fragen, welchen Zweck eigentlich die ubergrofse Menge des Alkohols bat. Denn angenommen, die Glyoxylsäure entstände aus Letzterem in theoretischer Ausbeute durch Oxydation, so würden auf ein Molekül Alkohol immerhin zwei Molektile Salpetersaure erforderlich sein gemäfs der Gleichung :

$$
\mathrm{C}_{2} \mathrm{H}_{6} \mathrm{O}+\mathrm{N}_{2} \mathrm{O}_{5}+\mathrm{H}_{2} \mathrm{O}=\mathrm{C}_{2} \mathrm{H}_{8} \mathrm{O}_{3}+3 \mathrm{H}_{2} \mathrm{O}+\mathrm{N}_{2} \mathrm{O} \text {. }
$$

Dieselbe Menge Salpetersăure würde nötig sein, wenn an Stelle der Glyoxylsaure Oxalsäure erzeugt wirde, in dem Falle müfste dio Salpetersäure bis zu Stickstoff reduziert werden. Nun entstehen in dem Prozesse neben vorgenannten Verbindungen noch Essigsäure, Glycolsäure und Glyoxal, deren Erzeugung bei weitem weniger Sauerstoff orfordert. Acetaldehyd konnte ich unter den Oxydationsprodukten nicht auffinden, ich vermute seine Bildung 
in dem Prozesse, nicht darum, weil, wie ich gefunden habe, aus Acetaldehyd Glyoxylsäure gewonnen werden kann, (Ber. d. d. chem. Ges. XI. 1784) sondern weil ich seinen Geruch hin und wieder wabrgenommen habe. Auch die von Debus gefundene Ameisensäure vermochte ich unter den von mir innegehaltenen Versuchsbedingungen nicht nachzuweisen. Dagegen konstatierte ich die reichliche Bildung von Aethylnitrit, welches sich in Folge seines niedrigen Siedepunktes, unterstatzt von der audauernden Gasentwicklung, verfluchtigt und hierdurch eine Quelle bedeutenden Verlustes darstellt, wenn nicht für Kondensation Sorge getragen wird. Bei niedriger Temperatur sondert sich eine Aethylnitrit reiche Schicht auf der Oberfläche der Flüssigkeit ab, welche abgehoben werden kann. Nach dem Verseifen des Aethylnitrits mit reinem Kali läfst sich die Salpetrigesäure durch die gewöhnlichen Reagentien nachweisen. Das Aethylnitrat scheidet auf Zusatz von Phenylhydrazin und Essigsäure ein charakteristisch riechendes Oel $a b$, verhält sich also diesbezüglich ähnlich der Salpetrigensäure. Sein Verhalten bedingt, dals sich die Entwicklung des Prozesses nicht mittelst Phenylhydrazin kontrolieren lälst.

Beim Operieren in niedriger Temperatur kann die Abscheidung langer, farbloser, von Gasblasen über der grünen Zone schwebend gehaltener Nadeln beobachtet werden. Diese Nadeln erwiesen sich bei näherer Untersuchung als Oxalsäure.

Werden die zur Darstellung der Glyoxylsäure dienenden Ausgangsmaterialien in genügend kaltem Zustande angewendet, so erfolgt die Vermischung auch dann ausserordentlich träge, wenn dio Gefälse in einem tagsüber geheizten, frostfreien Zimmer stehen. Noch nach vier Wochen kann die Bodenschicht aus gelber Salpetersäure bestehen, ein brüskes Vermischen fthhrt auch dann zu gewaltsamer, Verlust bringender Reaktion. Hat sich die Vermischung vollzogen, so empfiehlt es sich, wie anch $D$ e b u s angiebt, die Flussigkeit noch einige Tage in der Nähe des Ofens, d. h. bis zum Aufhören der Gasentwicklung stehen zu lassen. Die Salpetersäure ist dann nahezu aufgebraucht und die Weiterverarbeitung der Mischung hat keine heftigere Nachoxydation im Gefolge. Sollte eine solche nach dem Concentrieren doch eintreten, so lürst sich ihre Entwicklung durch Zutröpfeln von etwas Alkohol unterdrücken. Die 
Weiterverarbeitung der Flüssigkeit geschieht nun so, dafs dieselbe mit Anwendung eines Wasserbades zunächst aus einer tubulierten Retorte destilliert wird. Durch den Tubulus geht die Trichterröhre eines Scheidetrichters, der mit Portionen der Flussigkeit gefüllt wird. Rasch destilliert Aethylnitrit ab. Dasselbe sammelt sich auf dem langsam abdestillierenden, lediglich Essigsäure enthaltenden, darum ammoniakalische Silberlossung nicht reduzierenden Wasser. Aethylnitrit und saures Wasser werden inittelst des Scheidetrichters von einander getrennt, und Frsteres bei erneuter Darstellung der Glyoxylsăure dem Alkohol beigemischt.

Beim Neutralisieren der stark gewässerten Essigsăure mic kohlensaurem Kalk wird noch etwas Aethylnitrit abgeschieden, welches gleichfalls in der angegebenen Weise Verwendung findet. Das gewonnene Calciumacetat besitzt die bekannten Eigenschaften.

$0.4007 \mathrm{~g}$ Salz lieferten $0.1413 \mathrm{~g}$ Kalk oder $25,19 \%$ Ca. Berechnet $\left(\mathrm{C}_{t} \mathrm{H}_{8} \mathrm{O}_{\mathrm{g}}\right)^{2} \mathrm{Ca}$ fur $\mathrm{Ca}: 25,32 \%$.

Die vorgenannten Resultate werden gewonnen, wenn die $D$ e b u s . sche Vorschrift folgende Fassung erhïlt, welche eine bedeutende Alkoholersparnis zur Folge hat:

$\mathrm{Je} 100 \mathrm{Cbc}$ nicht tuber $5^{\circ} \mathrm{C}$ warme Salpetersaure von $1.48 \mathrm{spec}$. Gew. werden in $44 \mathrm{Ctm}$. hohen Cylindern mit Hülfe einer G a y L ussa c Bürette mit 27 Cbc Wasser vorsichtig bedeckt, dann werden $50 \mathrm{Cbc}$ Alkohol abs. ebenso vorsichtig eingetragen. Wasser und Alkohol werden an dem Gefälsrand niederfliessen gelassen, was besonders leicht nnd ganz gefahrlos gelingt, wenn die Cylinder 7 Ctm. vom oberen Rande ab ausgebuchtet sind. Die Cylinder werden mit Uhrgläsern bedeckt. An die Stelle der Einschnürung wird ein kleines Uhrglas gebracht, welches im Laufe der Operation hin und wieder mit etwas kaltem Alkohol bedeckt wird. Treten uber der Flüssigkeit rote Dumpfe auf, so beseitigt man sie, indem man an der Cylinderwand einen Tropfen Alkohol niederfliefsen läfst.

Bezuglich der weiteren Verarbeitung verweise ich auch auf meine Abhandlung: Beitrag zur Kenntnis der Glyoxylskure, Liebigs Annalen 198, 206. Aufser dem daselbst erwăhnten Kalksalz von der Zusammensetzung $\left(\mathrm{C}_{2} \mathrm{H}_{3} \mathrm{O}_{4}\right)_{2} \mathrm{Ca}$ vermag die Glyoxylsäure noch ein wasserreicheres Salz zu bilden. Dieses scheidet sich aus der kalten verdünten Lösung allmählich aus. Es besitzt die Zusammensetzung 
$\left(\mathrm{C}_{2} \mathrm{H}_{3} \mathrm{O}_{4}\right)_{2} \mathrm{Ca}+2 \mathrm{H}_{8} \mathrm{O}$ und verliert das beigeschriebene Wasser hei kurzem Erhitzen auf $110^{\circ}$

$0,242 \mathrm{~g}$ lufttrockenes Salz lleferten $0,0528 \mathrm{~g}$ Kalk oder $15.58 \% \mathrm{Ca}$. Berechnet für obige Formel: $15.5 \%$ Ca.

Die Glyoxylsäure ist mit Wasserdämpfen, namentlich in konzentrierter Lösung stark flüchtig und riecht erstickend. Sie liefert bekanntlich ein schön krystallisierendes Oxim, wenn ihre mit Soda neutralisierte Lösung mit salzsaurem Hydroxylamin kurze Zeit auf dem Wasserbade erhitzt wird. Auf diese Weise wenigstens habe ich fragliche Verbindung unmittelbar nach dem Bekanntwerden der Reaktion dargestellt und Herrn Prof. V. Meyer in Göttingen privatim darüber berichtet. Die Glyoxylsaure liefert ein charakteristisches Hydrazon. Es entsteht, wenn die wässrige Lösung der Säure mit Phenylhydrazin versetzt wird. Beim Uebergielsen des auf einer Uhrschale ausgebreiteten Hydrazons mit koncentrierter Schwefelsäure bilden sich um die einzelnen Partikelchen braunrote Höfe und die Schwefelsänre wird von blaugrünen Schlieren durchzogen. Diese verschwinden keim Umschütteln, allmählich verblafst die Säure wieder. Wenn die wässrige Lösung des Calciumglyoxylats mit Phenylhydrazin geschüttelt wird, so entsteht nur eine gelbe Trübung und erst nach Zusatz von Essigsäure füllt ein Haufwerk feiner Nadeln aus. Dies Verbalten scheint anzudeuten, dals das Calciumglyoxylat das gebundene Wasser in besonderer Form enthalt und darum eine andere Constitution hat wio die freie Säure.

In dem beschriebenen Prozers entsteht bekanntlich neben Glyoxylsk̈ure stets Glycolsäure. Die Kalksalze beider Säuren lafsen sich in Folge ihrer verschiedenen Löslichkeit in Wasser unschwer von einander trennen, wenn sie mit einander vermischt werden. Bei der gemeinsamen Erzeugung der beiden Sauren in dem besprochenen Prozesse verestert sich eine gewisse Menge derselben zu einem durch andauernde Behandlung mit Wasser allmählich aufspaltbaren Produkt, welches nach der Neutralisation mit Kreide ein in Wasser sehr leicht lösliches, zersetzliches, durch Alkohol spaltbares Salz liefert, welches nicht in Krystallen zu gewinnen ist. Einer solcher Veresterung ist die Glyoxylsäure allein fähig, das Produkt derselben liefert ein Kalksalz von den eben beschriebenen Eigenschaften. Diese Salze müssen mittelst Alkohol von dem 
Glyoxal getrennt werden. In Folge ihrer Zersetzlichkeit verunreinigen die Spaltungsprodukte das Glyoxal. Werden die Salzlösungen verdampft, so nehmen sie saure Reaktion an und es verfluchtigt sich Glyoxylsurure, welche an dem erstickenden, dem Formaldehyd ähulichen Geruch kenntlich ist. Dem Verdampfungsrückstand kann mittelst Alkohol freie Glyoxylsäure entzogen werden. Das zurückbleibende Kalksalz liefert im exsiccatortrocknen Zustand verglüht die $9,94 \%$ Calcium entsprechende Kalkmenge. - Zufallig verlangt ein $\mathrm{Salz}$ von der Zusammensetzung $\mathrm{C}_{2} \mathrm{H}_{3}$ ca $\mathrm{O}_{4}+\mathrm{C}_{2} \mathrm{H}_{4} \mathrm{O}_{4}$ 9,85\% Ca. Darnach scheint der bimolekularen Form eine gröfsere Beständigkeit innezuwohmen, wie der höher (tri?) molekularen Form.

Wird Kalkbrei in die kalte wässrige Losung dieser Salzgemische eingetragen, so scheidet sich unter beträchtlicher Temperaturerhöhung eine Salzmasse aus, welche durch Wasser in wenig Calciumglycolat und viel Calciumglyoxylat zerlegt wird. Die Benutzung des Phenylhydrazins leistet bei dieser Diagnose bessere Dienste wie die Analyse, denn die Glycolsäure reagiert mit Phenylhydrazin nicht wie die Glyoxylsâure. Der von mir in der oben citirten Abhandlung empfohlene Wasserzusatz ist sonach zu ersetzen.

Uebrigens liefert die Glyoxylsaure beim Kochen z. B. mit Essigsäureanhydrid ein klebriges, zähes Acetat, welches von kaltem Wasser, in welchem es schwer löslich ist, sehr allmählich in Glyoxylslure und Essigsture aufgespalten wird, wie sich mittelst Phenylhydrazin nachweisen lăsst. Die wässrige Lösung verlangt zur Neutralisation nur wenig Calciumcarbonat, das erzielte Kalksalz krystallisiert nicht. Seine Lösung säuert beim Eindampfen nach, das mit Alkohol gefällte Salz reagiert ebenfalls sauer.

Darmstadt, 13. Januar 1894. Chem. Tech. Lab. (Privat). 